\title{
OBSERVACIONES SOBRE LOS CRITERIOS NORMATIVOS PARA ESTIMAR DESPLAZAMIENTOS INELÁSTICOS EN EDIFICIOS A BASE DE MARCOS UBICADOS EN TERRENO BLANDO
}

\author{
Jorge Ruiz García ${ }^{(1)}$ y Erick Jafet González Cuevas ${ }^{(2)}$
}

\begin{abstract}
RESUMEN
Se presentan los resultados de la evaluación de los criterios normativos incluidos en las NTC-Sismo edición 2004 para estimar desplazamientos laterales en edificios. Para tal fin, se evaluaron las demandas de desplazamiento máximo elástico e inelástico en 10 edificios estructurados a base de marcos resistentes a momento por medio de análisis dinámicos lineales y no-lineales. Los resultados indican que existe una zona espectral donde las distorsiones elásticas son mayores que las distorsiones inelásticas, lo cual no se reconoce en los criterios normativos para estimar desplazamientos. Asimismo, se muestra que el criterio para estimar desplazamientos laterales en condiciones de servicio incluido en el Apéndice A proporciona estimaciones razonables.
\end{abstract}

Palabras clave: desplazamiento inelástico; desplazamiento elástico; terreno blando; factor de amplificación de desplazamiento

\section{OBSERVATIONS ABOUT THE CODE CRITERIA FOR ESTIMATING INELASTIC DISPLACEMENTS IN FRAMED BUILDINGS LOCATED ON SOFT SOIL SITES}

\begin{abstract}
This paper presents the results of evaluating the 2004 seismic code criteria to estimate lateral displacements in buildings. For this purpose, the elastic and inelastic displacement demands in 10 buildings having moment resisting frames as lateral structural system through rigorous nonlinear time history analyses. Results show that there is a spectral region where elastic displacement demands are larger than inelastic displacement demands which is not recognized in the 2004 seismic code criteria. It is also shown that the current criterion for estimating lateral displacements for service conditions included in Appendix A leads to reasonable estimations.
\end{abstract}

Keywords: Inelastic displacement; elastic displacement; soft soil site; displacement amplification factor

Artículo recibido el 9 de octubre de 2015 y aprobado para su publicación el 4 de abril de 2016. Se aceptarán comentarios y/o discusiones hasta cinco meses después de su publicación.

(1) Profesor-Investigador Titular, Facultad de Ingeniería Civil, Universidad Michoacana de San Nicolás de Hidalgo, Edificio C, Cd. Universitaria, 58040, Morelia, Mich.; jruizgar@stanfordalumni.org

(2) Egresado de la Maestría en Estructuras, Facultad de Ingeniería Civil, Universidad Michoacana de San Nicolás de Hidalgo, Edificio C, Cd. Universitaria, 58040, Morelia, Mich. 


\section{INTRODUCCIÓN}

Los criterios de diseño sísmico adoptados por los códigos de construcción modernos tienen como objetivo principal: 1) evitar el daño tanto estructural como no-estructural ante eventos sísmicos que ocurran frecuentemente durante la vida útil de la estructura; 2) limitar el daño estructural del sistema ante sismos moderados que puedan presentarse con una probabilidad significativa durante el lapso de funcionamiento de la estructura, y 3) permitir daño estructural si se presentara un sismo excepcionalmente severo, pero de ninguna manera llegar al colapso de la estructura (Bazán y Meli, 1999). Para fines de diseño sísmico, la estimación del desplazamiento máximo es un parámetro muy importante en la respuesta sísmica de un edificio, ya que puede relacionarse con el nivel de daño en elementos estructurales y, en algunos casos, con el daño en elementos no estructurales que pueden presentarse en un edificio durante un evento sísmico. Por tal motivo, es deseable que este desplazamiento máximo sea estimado de manera razonable, teniendo en cuenta todas las incertidumbres involucradas en el proceso de diseño y la excitación.

Los códigos de diseño sísmico a nivel mundial estiman los desplazamientos máximos inelásticos que un edificio pudiera exhibir ante un evento sísmico al multiplicar los desplazamientos laterales de diseño obtenidos mediante un análisis elástico (empleando el método estático, con fuerzas de diseño reducidas, o el método modal espectral) por un factor adimensional. Por ejemplo, la normatividad americana denomina a este factor como el factor de amplificación de desplazamientos $C_{d}$, el cual depende del tipo de sistema estructural empleado (Uang 1991; Uang y Maarouf, 1994; Carrillo et al., 2014). Considerando una respuesta global fuerza lateral-desplazamiento bilineal de un sistema estructural, Uang (1991) demostró que $C_{d}$ es igual al factor de ductilidad de desplazamiento multiplicado por un factor de sobrerresistencia. Asimismo, Uang y Maarouf (1994) notaron que el cociente de $C_{d}$ y el factor de reducción de fuerzas por ductilidad (denominado $R$ en la normatividad americana, $q$ en el código europeo, o $Q$ ' en la normatividad Mexicana) es igual al cociente del desplazamiento máximo inelástico, $\Delta_{i}$, y el desplazamiento máximo elástico, $\Delta_{e}$, del sistema estructural. Con el objetivo de evaluar el cociente $C_{d} / R$, o bien $\Delta_{i} / \Delta_{e}$, Uang y Maarouf (1994) desarrollaron un estudio considerando cuatro edificios con diferente número de niveles (dos de concreto reforzado, de 6 y 10 niveles, y dos de acero, de 2 y 13 niveles) sujetos ante un conjunto de 10 registros históricos (incluyendo el acelerograma registrado en la estación SCT durante el terremoto de Michoacán de 1985). Entre sus conclusiones, los autores observaron que el cociente $C_{d} / R$ (o bien $\Delta_{i} / \Delta_{e}$ ) puede exceder significativamente el valor de uno para relaciones $T / T_{g}$ (donde $T$ es el periodo fundamental del edificio y $T_{g}$ es el periodo dominante del movimiento del terreno) menores a 0.3, y varía entre 0.7-0.9 para relaciones $T / T_{g}$ mayores que 0.3. Estos resultados implican que los desplazamientos máximos inelásticos sólo son significativamente mayores que los desplazamientos máximos elásticos para edificios con periodos de vibración significativamente más largos que el periodo dominante del movimiento del terreno.

Estudios realizados en sistemas de un grado de libertad, S1GL, sujetos a historias de aceleración del terreno registradas en terreno blando (Miranda, 1991; Santa Ana, 1994; Miranda y Ruiz, 1999; Espinoza y Terán, 2000; Ruiz-García y Miranda, 2004; 2006) han mostrado que la distribución espectral del cociente $\Delta_{i} / \Delta_{e}$ no es constante, y se pueden distinguir tres regiones espectrales. Por ejemplo, Ruiz-García y Miranda $(2004,2006)$ notaron que en la primera región, las ordenadas del cociente $\Delta_{i} / \Delta_{e}$ se incrementan conforme $T / T_{g}$ decrece. En la segunda región, para valores de $T$ entre $0.75 T_{g}$ y $1.5 T_{g}$, las ordenadas del cociente $\Delta_{i} / \Delta_{e}$ son menores que uno, lo cual implica que los desplazamientos máximos inelásticos son menores que los desplazamientos máximos elásticos. Finalmente, en la tercera región espectral, para valores de $T$ mayores que $1.5 T_{g}$, el cociente $\Delta_{i} / \Delta_{e}$ tiende a la unidad. Desafortunadamente, las tendencias descritas anteriormente no fueron corroboradas en estudios basados en sistemas de múltiples grados de libertad, como el estudio desarrollado por Uang y Maarouf (1994).

El objetivo general del trabajo de investigación que se presenta consistió en evaluar los criterios normativos incluidos en las Normas Técnicas Complementarias para Diseño por Sismo edición 2004, 
NTCS-04 (NTCS, 2004) para estimar desplazamientos laterales en edificios estructurados a base de marcos ubicados en terreno blando. Para tal fin, se procedió a: 1) evaluar estadísticamente el cociente $\Delta_{i} / \Delta_{e}$ en sistemas de múltiples grados de libertad, y 2) comparar los desplazamientos laterales calculados con los criterios normativos establecidos en el Cuerpo y el Apéndice A de las NTCS-04 con los desplazamientos laterales calculados con análisis dinámicos. Cabe notar que en este estudio sólo se consideró el método estático de análisis para la evaluación de los desplazamientos. Esto se atribuye a que los criterios normativos para la obtención de desplazamientos laterales son aplicables tanto para el método estático como el método modal (sección 1.8 de las NTCS-04). Asimismo, exhibir la diferencia cuantitativa en el uso del método estático o dinámico modal para estimar desplazamientos laterales esta fuera del alcance de este estudio. Como parte de esta investigación, se evaluaron las demandas de desplazamiento máximo elástico e inelástico en modelos analíticos bidimensionales de 10 edificios con diferente número de niveles y estructurados a base de marcos resistentes a momento, 4 edificios de acero y 6 edificios de concreto reforzado, por medio de análisis dinámicos lineales y no-lineales. Sin embargo, cabe notar que los modelos analíticos no consideraron la influencia de la interacción suelo-estructura.

\section{ESTIMACIÓN DESPLAZAMIENTOS INELÁSTICOS DE ACUERDO AL RCDF-2004}

La filosofía de diseño sismorresistente inherente en las NTCS-04 considera que las estructuras (por ejemplo, edificios) incursionarán en el intervalo de comportamiento inelástico ante sismos moderados e intensos. Por ello, para fines de diseño, el método estático de análisis y el método dinámico modal permiten reducir las fuerzas sísmicas (es decir, fuerzas laterales estáticas equivalentes) por medio del factor de reducción, $Q$ '. El valor del factor $Q^{\prime}$ es función del periodo fundamental de la estructura, $T$, del periodo característico, $T_{a}$, y del factor de comportamiento sísmico $Q$, el cual depende del tipo de sistema estructural que resiste las fuerzas laterales y de los detalles de dimensionamiento, adoptando valores entre 1 y 4 . Una vez realizado el análisis estático o dinámico modal, las NTCS-04 requieren que se revisen las distorsiones (es decir, la diferencia relativa entre desplazamientos laterales de dos pisos consecutivos), los cuales deberán ser menores que ciertas distorsiones límite. En particular, la sección 1.8 del cuerpo del las NTCS-04 establece lo siguiente: "El desplazamiento será el que resulte del análisis con las fuerzas sísmicas reducidas según los criterios que se fijan en el Capitulo 4, multiplicado por el factor de comportamiento sísmico, Q". Además estipula que cuando se aplique el método de análisis del Apéndice A, se aplicarán los límites que ahí se establecen para los desplazamientos. De esta manera, el cuerpo de las NTCS-04 implícitamente proporciona el siguiente criterio para estimar los desplazamientos inelásticos:

$\Delta_{i}=Q \Delta_{e}$

donde $\Delta_{i}$ es el desplazamiento lateral inelástico, $\Delta_{e}$ es el desplazamiento lateral obtenido con un análisis elástico considerando las fuerzas sísmicas reducidas y $Q$ es el factor de comportamiento sísmico por el que se reducen las fuerzas. Tanto $\Delta_{i}$ como $\Delta_{e}$ son desplazamientos laterales medidos respecto a la base.

En el caso de que se aplique el método de análisis sísmico estático descrito en Apéndice A de las NTCS-04, la revisión de desplazamientos laterales se especifica en la sección A.4 de acuerdo a los siguientes criterios (Ordaz et al., 2003):

a) Para limitación de daños a elementos no estructurales, las diferencias entre los desplazamientos laterales de pisos consecutivos, multiplicadas por el factor $Q^{\prime} R / 7$ no excederán 0.002 o 0.004 , según sea el caso. Los valores de $Q$ ' y $R$ se calcularán para el periodo fundamental de la estructura.

b) Para seguridad contra colapso, las diferencias entre los desplazamientos laterales de pisos consecutivos multiplicadas por el factor $Q R$ y divididas por las diferencias de elevaciones 
correspondientes, no excederán las distorsiones de entrepiso establecidas en la tabla A.1 para los distintos sistemas estructurales.

Si bien, estas especificaciones están en términos de distorsiones, implícitamente involucran los desplazamientos laterales ya que:

$\delta=\frac{\Delta_{r}}{H}$

donde $\delta$ es la distorsión de entrepiso, definida como el desplazamiento relativo entre dos pisos sucesivos, $\Delta_{r}$, normalizado con respecto a la altura de entrepiso, $H$. Esto implica, por lo tanto, que los factores que se especifican en los incisos a) y b), de la sección A.4 del apéndice, son aplicables a los desplazamientos. De esta manera, los desplazamientos inelásticos se obtendrán como:

a) Para condiciones de servicio

$\Delta_{i}=\frac{1}{7} Q^{\prime} R \Delta_{e}$

b) Para seguridad contra colapso

$\Delta_{i}=Q R \Delta_{\mathrm{e}}$

donde $Q^{\prime}$ y $R$ son el factor de ductilidad y de sobrerresistencia, respectivamente, definidos en la sección A.3 del apéndice, referente a la definición de espectro para diseño sísmico. Estos factores son función del periodo fundamental de la estructura y los periodos característicos $T_{a}$ y $T_{b}$ del espectro de aceleraciones, que a su vez están en función del periodo dominante del sitio. Se calculan usando las siguientes expresiones:

$Q^{\prime}=\left\{\begin{array}{l}1+(Q-1) \sqrt{\frac{\beta}{k}} \frac{T}{T_{a}}, T \leq T_{a} \\ 1+(Q-1) \sqrt{\frac{\beta}{k}}, T T_{a}<T<T_{b} \\ 1+(Q-1) \sqrt{\frac{\beta p}{k}}, T>T_{b}\end{array}\right.$

donde $Q$ es el factor de comportamiento sísmico que se determina en el capítulo 5 del reglamento, $\beta$, es un factor de reducción por amortiguamiento suplementario, que es igual a uno cuando se ignora la interacción suelo-estructura, mientras que $k, T_{a}$ y $T_{b}$ son el coeficiente y los periodos característicos del espectro de aceleraciones, que se calculan con las expresiones descritas en el apartado A.3, los cuales son función del periodo dominante del terreno, $T_{s}$. Por otra parte, el factor de reducción por sobrerresistencia, $R$, se calcula con el siguiente criterio:

$$
R=\left\{\begin{array}{r}
\frac{10}{4+\sqrt{\frac{T}{T_{a}}},} T \leq T_{a} \\
2, T>T_{a}
\end{array}\right.
$$




\section{EDIFICIOS CONSIDERADOS EN ESTE ESTUDIO}

\section{Descripción}

Para fines de esta investigación, se consideraron dos grupos de edificios cuyo sistema resistente ante cargas laterales consiste de marcos resistentes a momento en ambas direcciones. Se asumió que los edificios están localizados en la zona del lago de la Ciudad de México, teniendo una distribución regular de masa, rigidez y resistencia en planta y altura. En el primer grupo, se consideraron seis edificios de concreto reforzado (CR) de 4, 6, 8, 10, 12 y 16 niveles, diseñados con los lineamientos del Reglamento de Construcciones del Distrito Federal Edición 1997 (Gaceta Oficial, 1997). En la figura 1 se muestra la planta típica de los edificios. En elevación, los edificios tienen una altura de $4.5 \mathrm{~m}$ en la planta baja y de $3.0 \mathrm{~m}$ en los niveles restantes. En su diseño, se consideró una resistencia nominal del concreto a compresión $f^{\prime}{ }^{\prime}=24.52$ $\operatorname{MPa}\left(250 \mathrm{~kg} / \mathrm{cm}^{2}\right)$ y un esfuerzo nominal de fluencia del acero de refuerzo $f_{y}=411.88 \mathrm{MPa}\left(4200 \mathrm{~kg} / \mathrm{cm}^{2}\right)$. Las fuerzas laterales fueron reducidas por un factor de comportamiento sísmico, $Q$, igual a 4 . Cabe notar que aun cuando estos edificios se diseñaron con un reglamento anterior a las NTCS-04, el criterio para calcular desplazamientos laterales es el mismo en ambos reglamentos. Una descripción detallada de la geometría y diseño de los edificios puede encontrarse en Terán (1998).

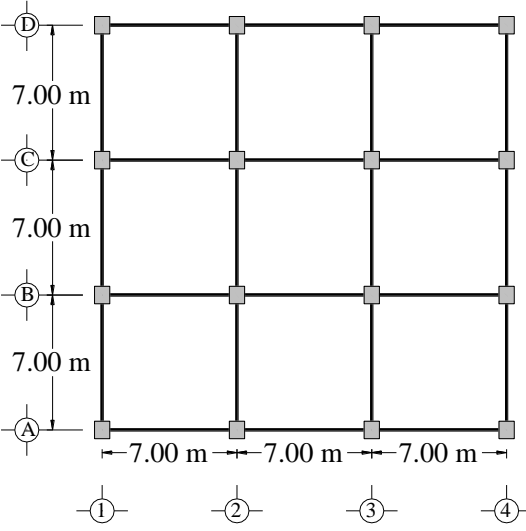

Figura 1. Planta típica de los edificios de concreto reforzado considerados en este estudio.

El segundo grupo incluyó un conjunto de 4 edificios de acero construidos comúnmente en la zona del lago del Valle de México. Los edificios fueron diseñados por Díaz (2006), bajo la supervisión de un despacho profesional. Para su diseño, se consideró que el uso de los edificios sería para oficinas (estructura tipo B) y que estaban ubicados en Zona IIIb (terreno blando) de la Ciudad de México de acuerdo a la zonificación indicada en las NTCS-04 del Reglamento de Construcciones del Distrito Federal vigente (Gaceta Oficial, 2004). En su diseño se consideró un factor $Q$ igual a 2, valor comúnmente utilizado en la práctica profesional. En la figura 2 se muestra la planta típica de los edificios. En elevación, los edificios tienen una altura típica de $3.5 \mathrm{~m}$. En su diseño, se consideró una resistencia nominal de fluencia del acero A-36, $F_{y}=248.11 \mathrm{MPa}\left(2530 \mathrm{~kg} / \mathrm{cm}^{2}\right)$. Una descripción detallada de la geometría y diseño de los edificios puede encontrarse en Díaz (2006).

\section{Modelado}

Los edificios seleccionados se modelaron bidimensionalmente y analizaron con el programa de computadora RUAUMOKO (Carr, 2009). Dada la simetría en planta, se consideró un marco exterior y un marco interior del sentido longitudinal, por lo que sólo se consideró la mitad del peso total del edificio, el cual se distribuyó por áreas tributarias y se concentró en los nodos del marco equivalente. Para fines de 
modelado de los edificios de CR y acero, el marco externo y el marco interno se colocaron en serie y se unieron con una trabe de liga articuladas en los extremos y con área muy grande comparada con la del resto de las secciones, con la finalidad de que los desplazamientos laterales fueran iguales en cada marco. El sistema de piso se modeló como un diafragma rígido. Las columnas se supusieron empotradas. En la figura 3 se ilustra esquemáticamente la estrategia de modelado de un edificio de 4 niveles.

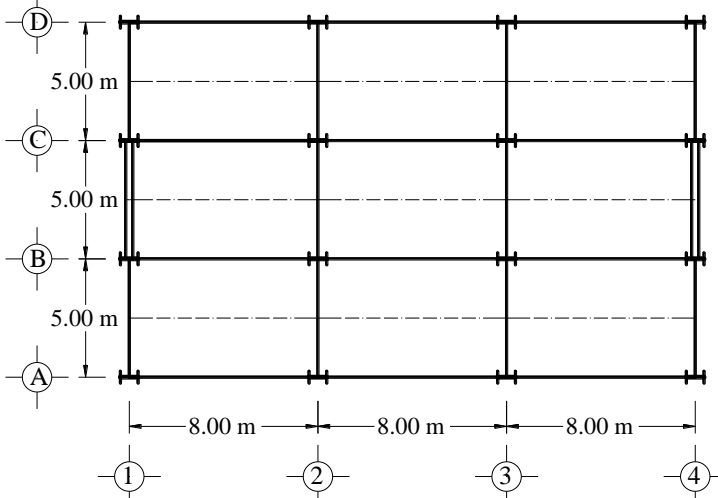

Figura 2. Planta típica de los edificios de acero considerados en este estudio.

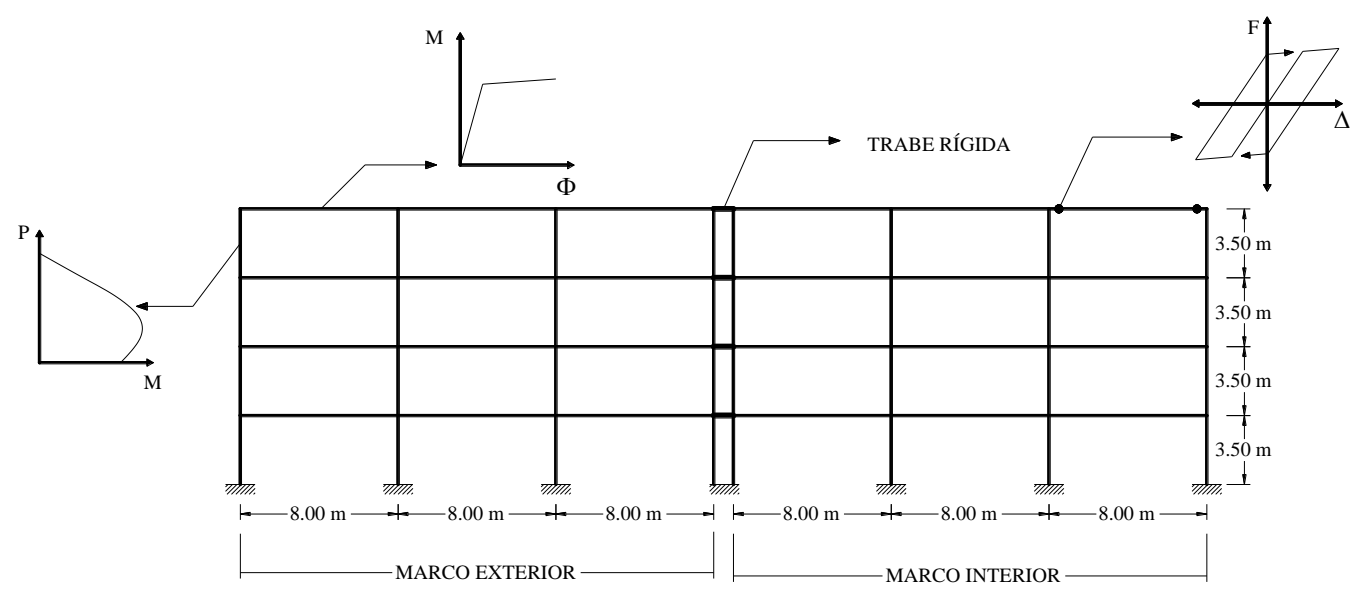

Figura 3. Estrategia de modelado de los edificios considerados en esta investigación.

Se consideró la formación de articulaciones plásticas en los extremos de vigas y columnas (es decir, criterio de plasticidad concentrada) ya que es donde se concentra el daño en estos elementos debido a las cargas cíclicas. Se supuso un comportamiento histerético momento-curvatura bilineal en los elementos de los edificios de acero, el cual asume que no se presentan problemas de inestabilidad local en el elemento. Para el caso de los elementos de CR, se supuso un comportamiento histerético momento-curvatura descrito por el modelo de Takeda, incluido en la biblioteca de RUAUMOKO (Carr, 2009) con parámetros $\beta=0 \mathrm{y}$ $\alpha=0.5$. Ambos modelos histeréticos consideraron una rigidez de post-fluencia igual a $0.01 \%$ para evitar problemas de inestabilidad numérica. En ambas familias de edificios se consideró explícitamente la interacción carga axial-momento en las columnas, de acuerdo al criterio incluido en el programa RUAUMOKO (Carr, 2009). Para fines del análisis dinámico, se consideró un amortiguamiento de Rayleigh, suponiendo un 5\% del amortiguamiento crítico en los primeros dos modos de vibración para los edificios de concreto, y del $3 \%$ del amortiguamiento crítico en los primeros dos modos de vibración para los edificios de acero. 
Antes de desarrollar análisis dinámicos, se llevaron a cabo análisis modal y estático no-lineales (denominados "pushover" en la literatura inglesa) para obtener las propiedades dinámicas y mecánicas de cada edificio. En las tablas 1 y 2 se indican dichas propiedades, donde $T_{l}$ es el periodo fundamental del edificio, $C_{y}$ se define como la fuerza cortante basal de fluencia normalizada respecto al peso total del edificio, $\theta_{y}$ es la distorsión de azotea asociada a la fluencia (es decir, el desplazamiento lateral de azotea asociado a la fluencia normalizado con respecto a la altura total del edificio).

Tabla 1. Propiedades de los marcos de acero

\begin{tabular}{cccc}
\hline MODELO & $T_{1}(\mathrm{~s})$ & $C_{y}$ & $\theta_{y}(\%)$ \\
\hline A-4N & 0.74 & 0.72 & 1.07 \\
A-6N & 0.88 & 0.65 & 0.95 \\
A-8N & 0.93 & 0.64 & 0.79 \\
A-10N & 1.06 & 0.59 & 0.74 \\
\hline
\end{tabular}

Tabla 2. Propiedades de los marcos de concreto reforzado

\begin{tabular}{cccc}
\hline MODELO & $T_{1}(\mathrm{~s})$ & $C_{y}$ & $\theta_{y}(\%)$ \\
\hline C- 4N & 0.81 & 0.32 & 0.54 \\
C- 6N & 1.14 & 0.22 & 0.51 \\
C- $8 \mathrm{~N}$ & 1.40 & 0.19 & 0.62 \\
C-10N & 1.40 & 0.19 & 0.54 \\
C-12N & 1.41 & 0.21 & 0.49 \\
C-16N & 1.74 & 0.20 & 0.58 \\
\hline
\end{tabular}

\section{EVALUACIÓN DE DESPLAZAMIENTOS}

\section{Registros sísmicos y escalamiento}

En este estudio se utilizó un conjunto de 20 acelerogramas registrados en estaciones ubicadas en terreno blando de la zona lacustre de la Ciudad de México. Los registros fueron obtenidos de la Base Mexicana de Sismos Fuertes (SMIS, 1999). Cada registro sísmico se corrigió por línea base y fue filtrado empleando el software Sismosignal (2016). En la tabla 3 se indican las características de cada uno de los registros, entre las que se incluyen el periodo predominante del terreno, $T_{g}$, el cual se calculó como el periodo asociado a la ordenada máxima en el espectro de velocidad, y la aceleración máxima del terreno, AMT.

Como puede apreciarse en la tabla 3, con excepción de los acelerogramas registrados durante el sismo del 19 de septiembre de 1985, los acelerogramas restantes fueron registrados durante sismos de moderada o baja intensidad, lo cual se refleja en el nivel de aceleraciones del terreno. En consecuencia, es de esperarse que los edificios no exhiban un comportamiento inelástico ante estas excitaciones y, por consiguiente, no inducirán demandas de desplazamiento inelástico. Por ello, en esta investigación se decidió escalar los registros sísmicos para asegurar que los edificios exhibieran demandas de desplazamiento inelástico. Para tal fin, en principio, se evalúo la resistencia lateral relativa de cada edificio, la cual se define como:

$$
R_{y}=\frac{\text { Demanda de resistencia lateral elástica }}{\text { Capacidad de fluencia de la estructura }}=\frac{S_{a}\left(T_{1}\right) / g}{C_{y}}
$$


donde $S_{a}\left(T_{1}\right)$ es la aceleración espectral asociada al periodo fundamental de la estructura correspondiente a un registro sísmico específico, $C_{y}$ es la fuerza cortante de fluencia normalizada respecto al peso de la estructura y $g$ es la aceleración de la gravedad. El coeficiente adimensional $C_{y}$ se obtuvo de un análisis estático no lineal de cada edificio. De acuerdo a la formulación, valores de $R_{y}$ menores a uno implican que el edificio no exhibe un comportamiento inelástico, mientras que valores mayores a uno indican que el edificio incurrió en comportamiento inelástico ante un registro específico. En la figura 4 se muestra la distribución de resistencias laterales relativas para cada familia de edificios. Como puede apreciarse, todos los edificios de acero se comportan elásticamente ante el conjunto de registros sísmicos, mientras que muy pocos registros inducen comportamiento inelástico en los edificios de concreto. Por ello, considerando que es deseable la predicción de desplazamientos máximos inelásticos, se procedió a escalar cada uno de los registros sísmicos, en amplitud, para que cada edificio alcance una resistencia lateral relativa igual a dos (es decir, cada historia de aceleraciones fue multiplicada por un factor mayor que uno para que las aceleraciones espectrales fueran mayores). De esta manera, el escalamiento de los acelerogramas garantizó la incursión en el intervalo inelástico de los marcos, lo cual no ocurría cuando se empleaban los registros sin escalar.

Tabla 3. Características del conjunto de acelerogramas considerados en este estudio

\begin{tabular}{cccccccc}
\hline \multirow{2}{*}{ Registro } & Estación & $\begin{array}{c}\text { Nombre } \\
\text { Estación }\end{array}$ & Fecha & $\begin{array}{c}\text { Magnitud } \\
(\mathrm{Ms})\end{array}$ & Componente & $\begin{array}{c}\text { AMT } \\
\left(\mathrm{cm} / \mathrm{s}^{2}\right)\end{array}$ & $\mathrm{T}_{\mathrm{g}}(\mathrm{s})$ \\
\hline SCT10DIEW & SC & SCT & $10 / 12 / 1994$ & 6.3 & EW & 15.0 & 1.89 \\
SCT19SEEW & SC & SCT & $19 / 09 / 1985$ & 8.1 & EW & 167.9 & 2.06 \\
SCT19SENS & SC & SCT & $19 / 07 / 1985$ & 8.1 & NS & 97.9 & 2.07 \\
4424OCEW & 44 & U. Colonia IMSS & $24 / 10 / 1993$ & 6.6 & EW & 15.0 & 1.34 \\
4424OCNS & 44 & U. Colonia IMSS & $24 / 10 / 1993$ & 6.6 & NS & 12.2 & 1.52 \\
5524OCNS & 55 & Tlatelolco & $24 / 10 / 1993$ & 6.6 & NS & 8.3 & 1.35 \\
RO14SEEW & RO & Roma & $14 / 09 / 1995$ & 7.1 & EW & 37.4 & 1.31 \\
RO14SENS & RO & Roma & $14 / 09 / 1995$ & 7.1 & NS & 28.6 & 1.40 \\
RO25ABEW & RO & Roma & $25 / 04 / 1989$ & 6.9 & EW & 54.7 & 1.27 \\
RO25ABNS & RO & Roma & $25 / 04 / 1989$ & 6.9 & NS & 45.4 & 1.53 \\
RO10DIEW & RO & Roma & $10 / 12 / 1994$ & 6.3 & EW & 12.0 & 1.39 \\
RO10DINS & RO & Roma & $10 / 12 / 1994$ & 6.3 & NS & 14.2 & 1.32 \\
4425ABEW & 44 & U. Colonia IMSS & $25 / 04 / 1989$ & 6.9 & EW & 39.6 & 1.28 \\
5325ABEW & 53 & San Simón & $25 / 04 / 1989$ & 6.9 & EW & 30.5 & 1.56 \\
4425ABNS & 44 & U. Colonia IMSS & $25 / 04 / 1989$ & 6.9 & NS & 52.3 & 1.36 \\
5325ABNS & 53 & San Simón & $25 / 04 / 1989$ & 6.9 & NS & 39.7 & 1.40 \\
2925ABEW & 29 & Villa del Mar & $25 / 04 / 1989$ & 6.9 & EW & 40.8 & 2.96 \\
2925ABNS & 29 & Villa del Mar & $25 / 04 / 1989$ & 6.9 & NS & 49.4 & 2.96 \\
4325ABNS & 43 & Jamaica & $25 / 04 / 1989$ & 6.9 & NS & 35.2 & 3.04 \\
4825ABEW & 48 & Rodolfo Menéndez & $25 / 04 / 1989$ & 6.9 & EW & 47.7 & 2.89 \\
\hline
\end{tabular}



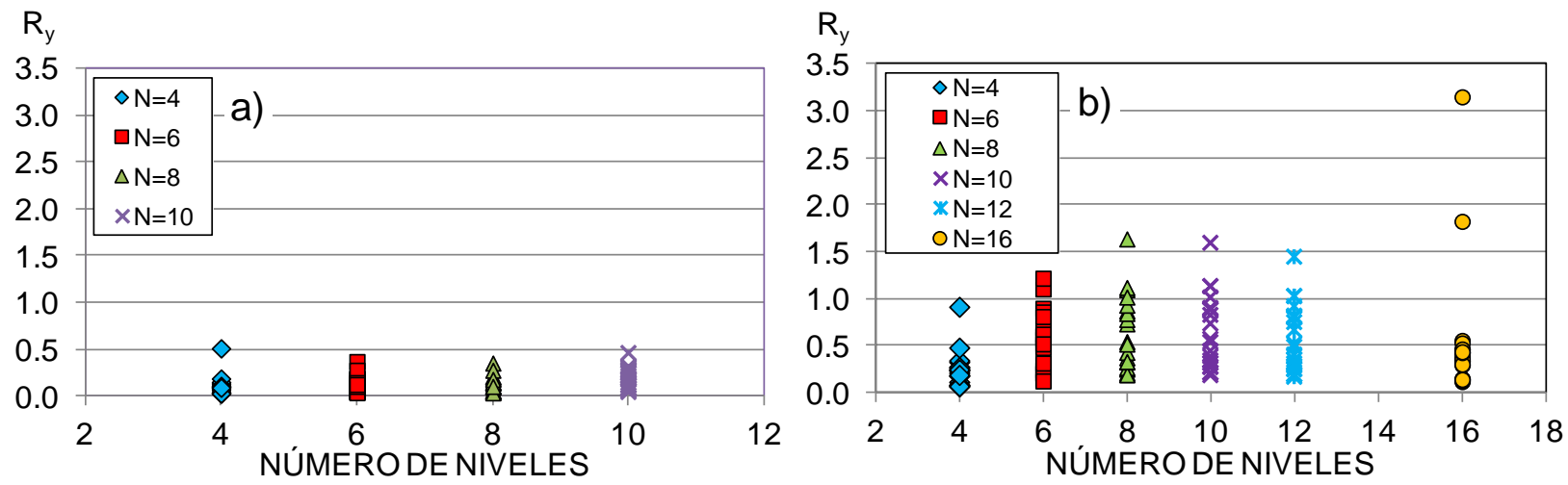

Figura 4. Resistencia lateral relativa, $R_{y}$, del conjunto de edificios calculada para el conjunto de acelerogramas: a) Edificios de acero, b) edificios de concreto reforzado.

\section{Demandas de desplazamiento inelástico}

En las figuras 5 a 8 se presentan ejemplos de perfiles de la demanda de desplazamiento máximo elástico e inelástico obtenidos para los marcos de acero de 4, 6, 8 y 10 niveles, cuando se sometieron a 3 de los 20 movimientos del terreno considerados. Note que cada registro sísmico tiene un periodo dominante, $T_{g}$, diferente y, en consecuencia, se obtienen tres diferentes relaciones $T / T_{g}$ para cada modelo.

En los perfiles de desplazamientos mostrados en las figuras 5 a 8 se puede observar que los desplazamientos inelásticos son mayores que los elásticos si la relación de periodos $T / T_{g}$ es pequeña. Sin embargo, cuando la relación $T / T_{g}$ crece, los desplazamientos inelásticos tienden a ser menores que los desplazamientos elásticos. Asimismo, se observa que la diferencia entre desplazamientos elásticos e inelásticos es grande cuando la relación $T / T_{g}$ disminuye, siendo el desplazamiento inelástico mayor que el elástico. Conforme aumenta la relación $T / T_{g}$, la diferencia entre ambos desplazamientos disminuye siendo los desplazamientos inelásticos muy parecidos a los elásticos, pero si la relación de periodos de la estructura y del movimiento sigue en aumento, los desplazamientos inelásticos decrecen, de tal manera que, incluso, llegan a ser menores que los elásticos.
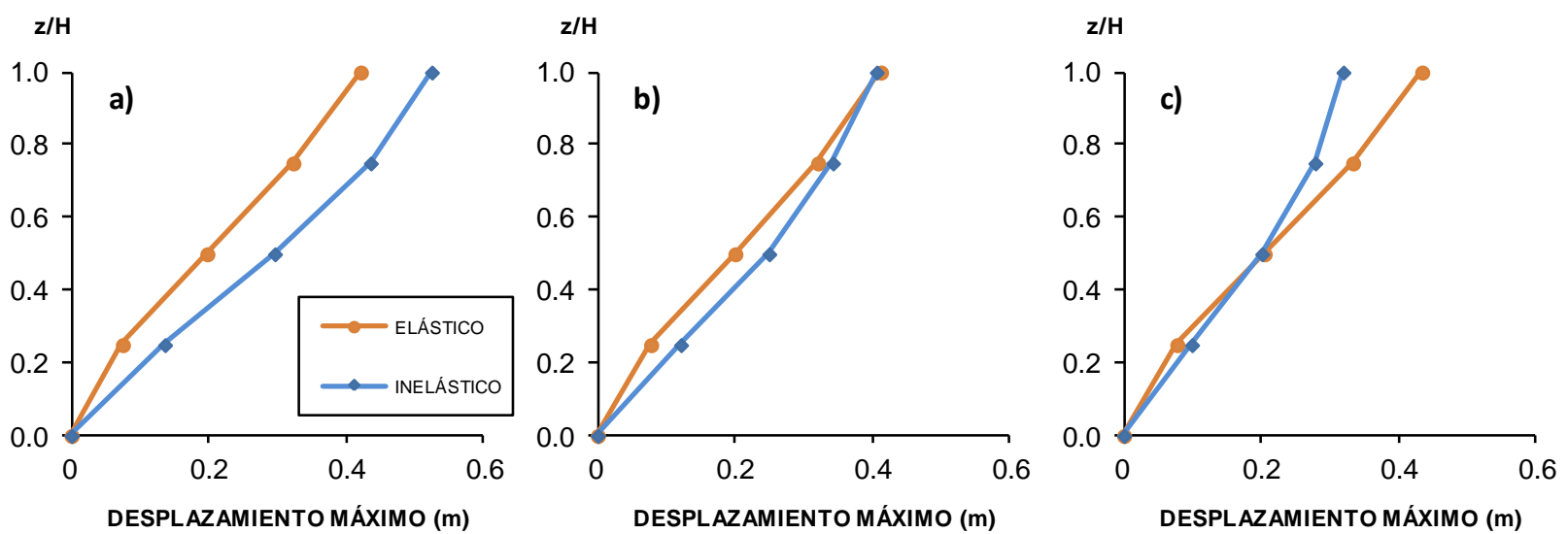

Figura 5. Perfiles de desplazamiento obtenidos para el modelo A-4N: a) $T / T_{g}=0.36$, b) $T / T_{g}=0.55$, c) $T / T_{g}=0.58$. 

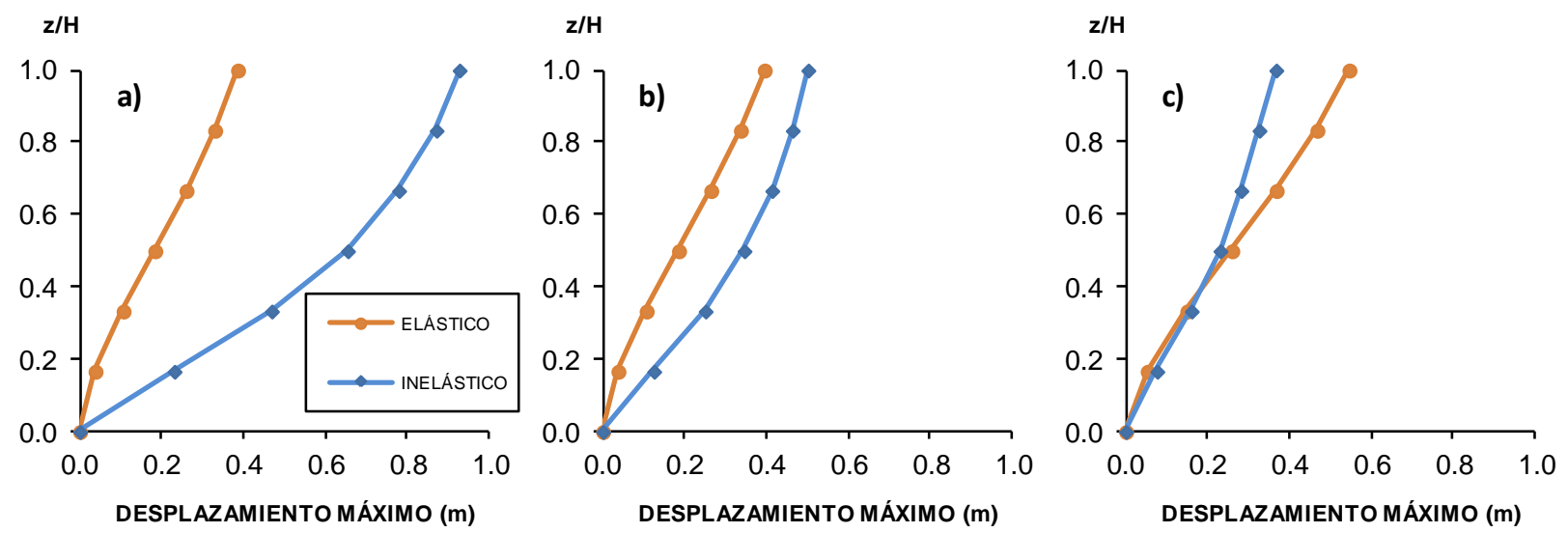

Figura 6. Perfiles de desplazamiento obtenidos para el modelo A-6N: a) $T / T_{g}=0.42$, b) $T / T_{g}=0.58$,
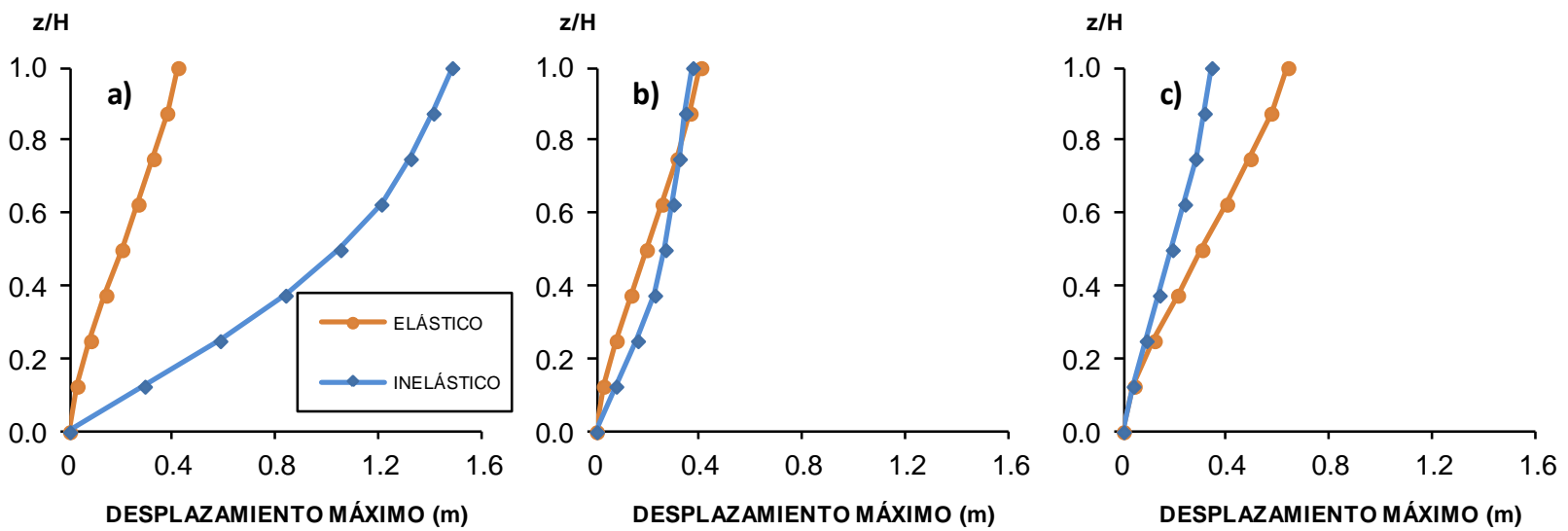

Figura 7. Perfiles de desplazamiento obtenidos para el modelo A-8N: a) $T / T_{g}=0.45$,

b) $T / T_{g}=0.61$, c) $T / T_{g}=0.72$.
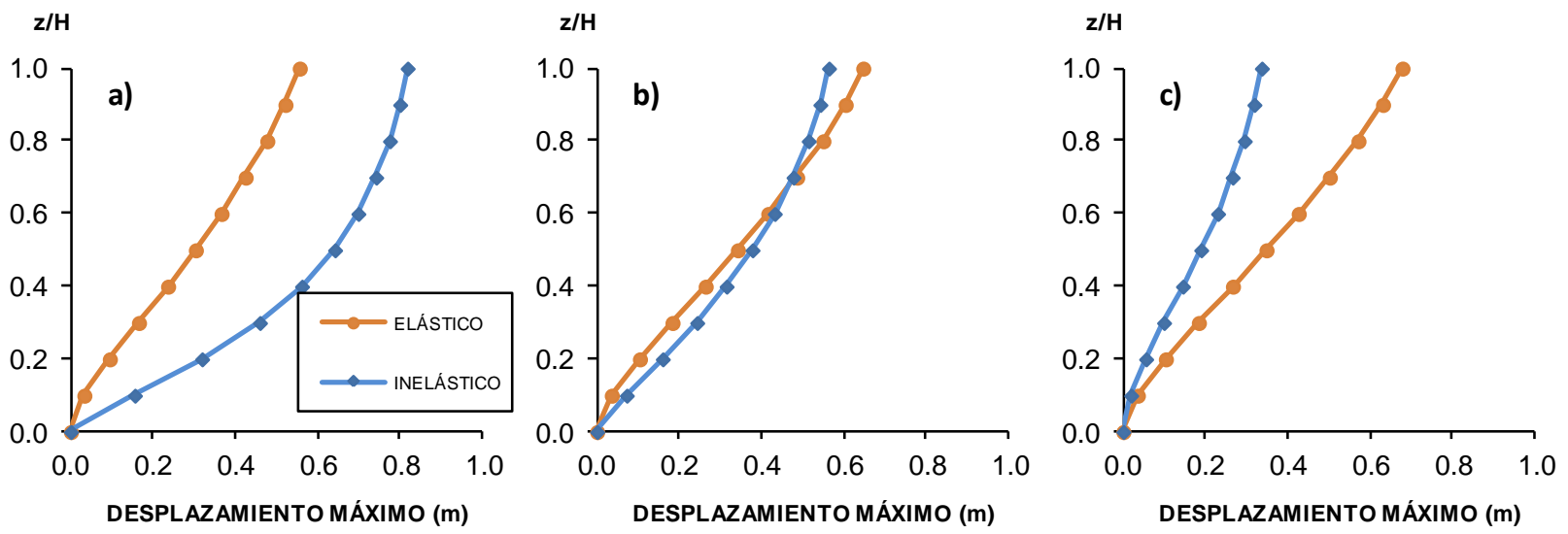

Figura 8. Perfiles de desplazamiento obtenidos para el modelo A-10N: a) $T / T_{g}=0.56$, b) $T / T_{g}=0.76$, c) $T / T_{g}=0.80$.

En las figuras 9 a 14 se presentan tres casos de perfiles de desplazamientos para diferente relación $T / T_{g}$, correspondiente a cada uno de los marcos de concreto de 4, 6, 8, 10, 12 y 16. En ellas se observa una tendencia similar que la mostrada en los edificios de acero, a excepción del modelo C-4N en el cual no se presenta ningún caso en el que los desplazamientos inelásticos sean menores que los elásticos. Lo anterior 
puede atribuirse a que las relaciones $T / T_{g}$ son menores que 0.75. Sin embargo, los edificios de acero presentan desplazamiento inelásticos menores que los elásticos, incluso para valores de $T / T_{g}$ pequeños.
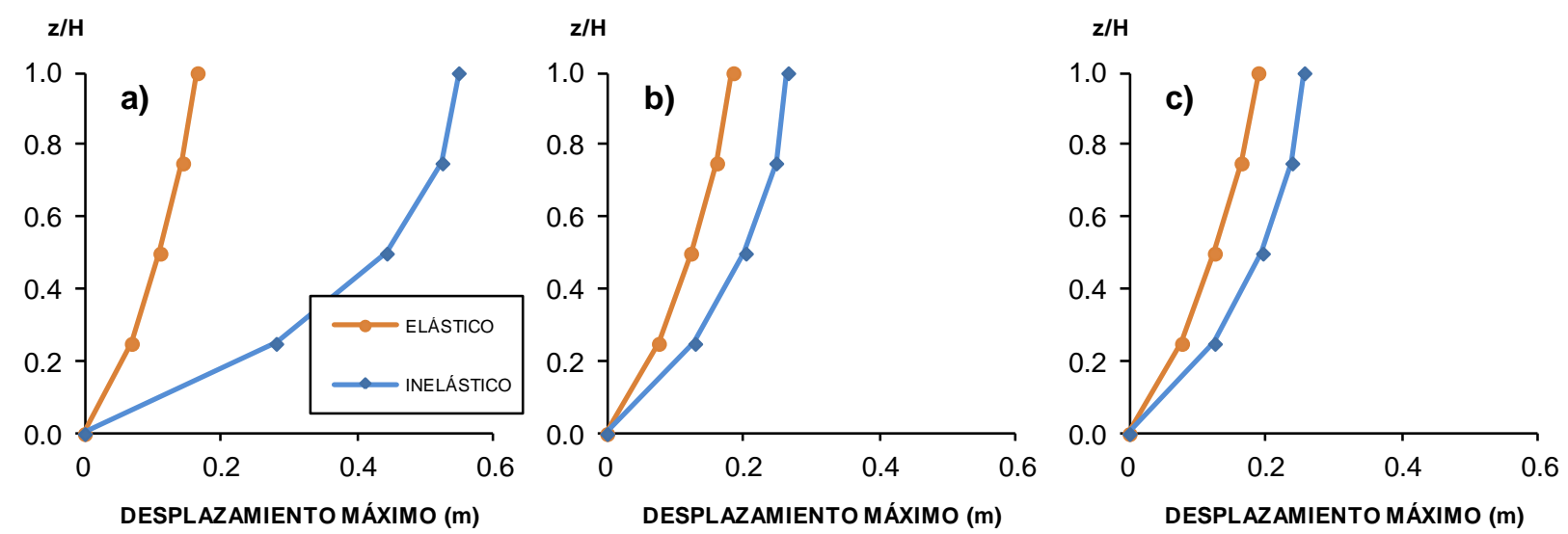

Figura 9. Perfiles de desplazamiento obtenidos para el modelo C-4N: a) $T / T_{g}=0.53$,
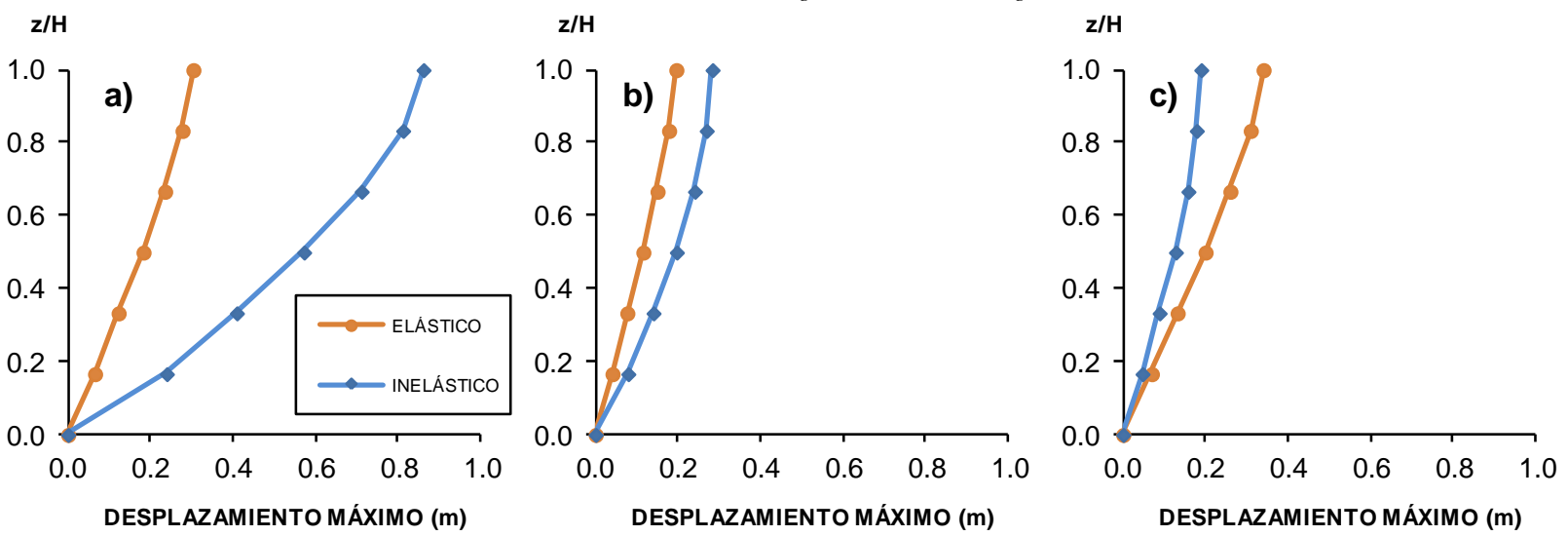

Figura 10. Perfiles de desplazamiento obtenidos para el modelo C-6N: a) $T / T_{g}=0.55$, b) $T / T_{g}=0.73$, c) $T / T_{g}=0.84$.
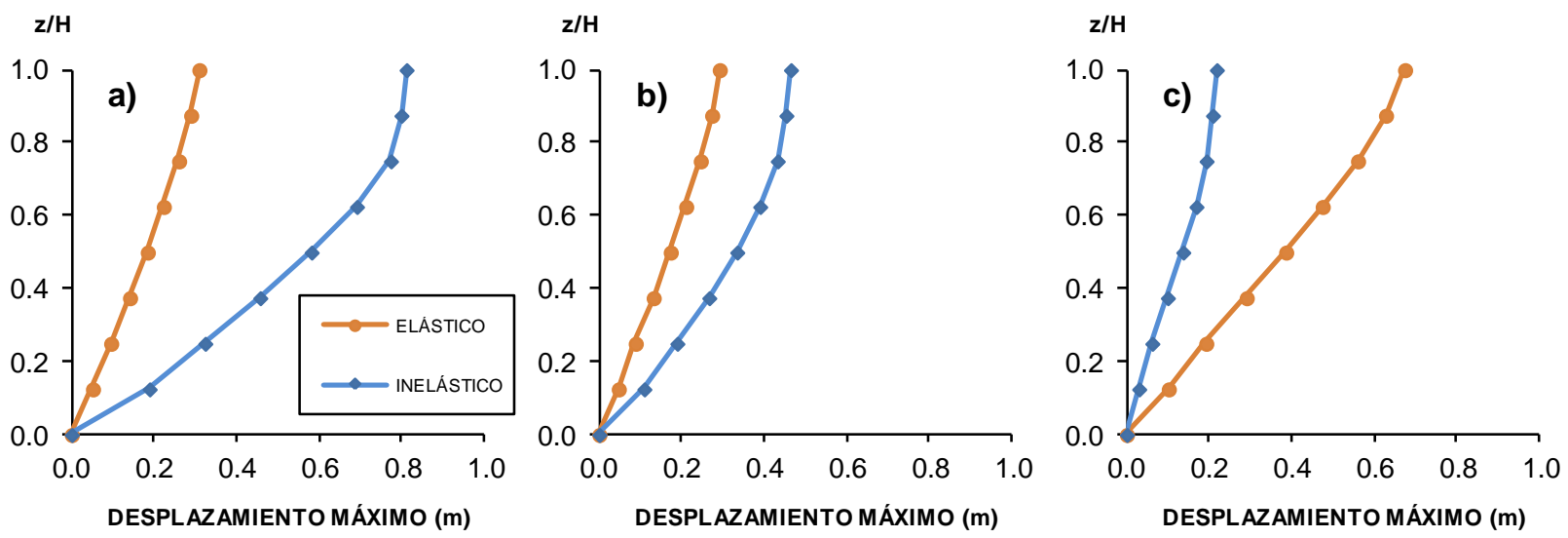

Figura 11. Perfiles de desplazamiento obtenidos para el modelo C-8N: a) $T / T_{g}=0.53$, b) $T / T_{g}=0.74$, c) $T / T_{g}=1.09$. 

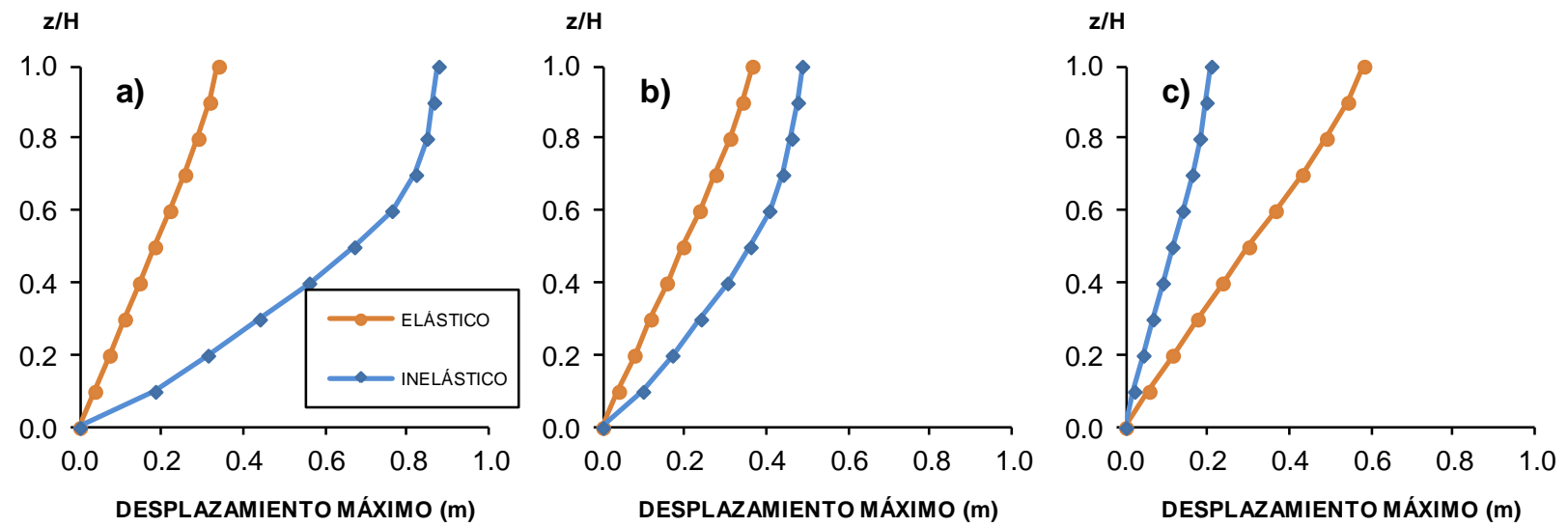

Figura 12. Perfiles de desplazamiento obtenidos para el modelo C-10N: a) $T / T_{g}=0.53$, b) $T / T_{g}=0.74$, c) $T / T_{g}=1.07$.
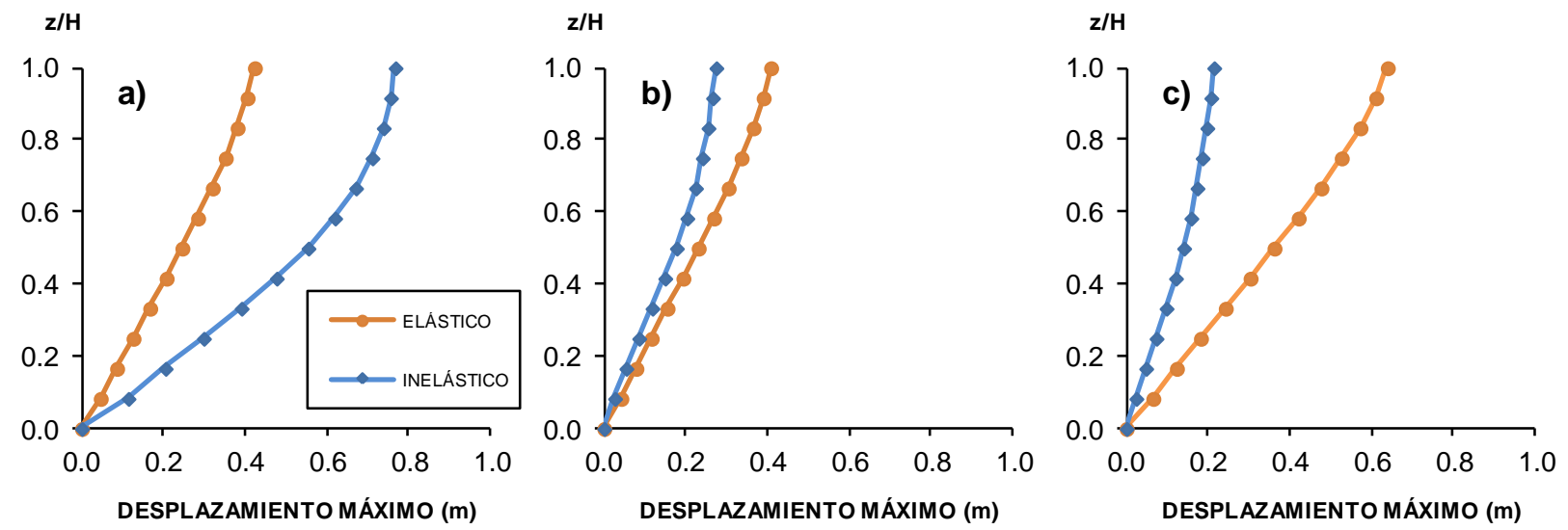

Figura 13. Perfiles de desplazamiento obtenidos para el modelo C-12N: a) $T / T_{g}=0.68$, b) $T / T_{g}=0.90$, c) $T / T_{g}=1.07$.
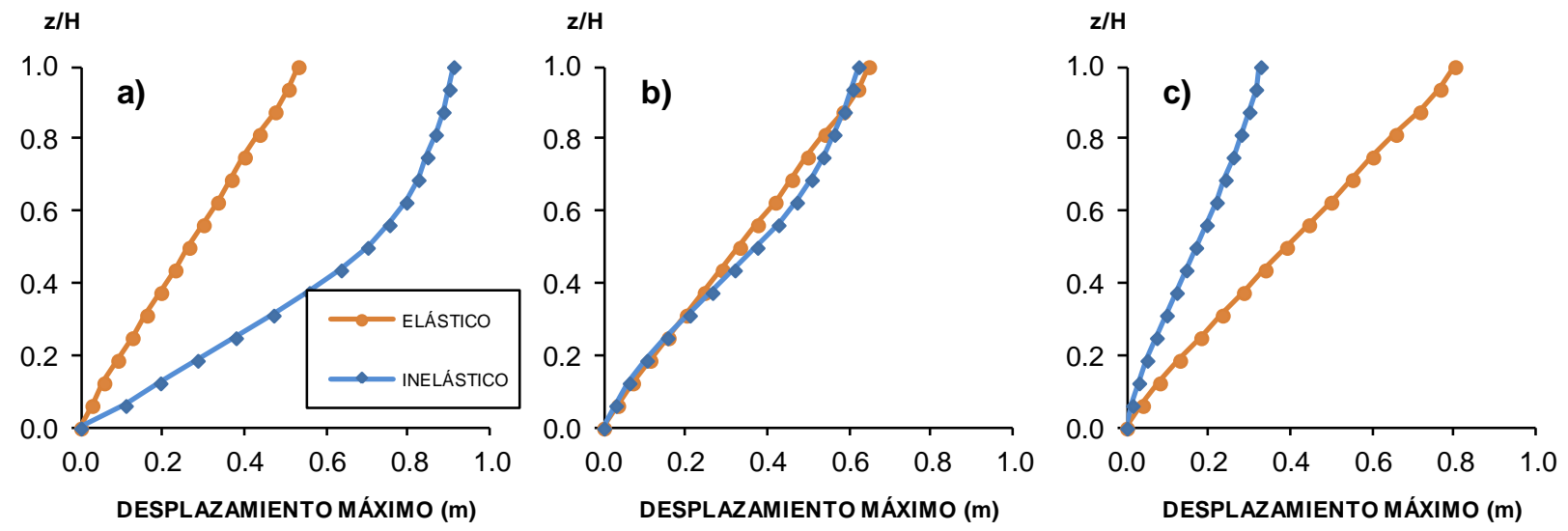

Figura 14. Perfiles de desplazamiento obtenidos para el modelo C-16N: a) $T / T_{g}=0.57$, b) $T / T_{g}=0.84$, c) $T / T_{g}=1.30$.

Con la finalidad de identificar una tendencia, en la figura 15 se muestra el cociente del desplazamiento máximo inelástico a desplazamiento máximo elástico de azotea, denominado $C_{M}$, obtenido para cada 
edificio sujeto a cada uno de los 20 acelerogramas incluidos en este estudio. Puede verse que la tendencia observada en los casos descritos anteriormente se conserva para cada familia de edificios. Es decir, existe una zona espectral $T / T_{g}$ donde los desplazamientos máximos inelásticos son mayores que los desplazamientos máximos elásticos, lo cual es consistente con los criterios normativos descritos anteriormente. Sin embargo, también puede apreciarse que existe una zona espectral $T / T_{g}$ donde los desplazamientos inelásticos son menores que los desplazamientos máximos elásticos, lo cual no está reconocido en los criterios normativos. Esta zona espectral depende del tipo de edificio (CR o acero). Es interesante notar que las tendencias descritas anteriormente fueron observadas en S1GL por Miranda y Ruiz (1999) y Ruiz-García y Miranda (2006), considerando S1GL con resistencia lateral constante, así como Miranda (1991), Santa Ana (1996), Ruiz-García y Miranda (2004), Espinoza y Terán (2000), considerando S1GL con ductilidad constante. De esta manera, los resultados obtenidos en esta investigación en sistemas de múltiples grados de libertad confirman las observaciones ofrecidas en estudios previos y deberían tenerse en cuenta para la estimación de desplazamientos inelásticos en terreno blando.
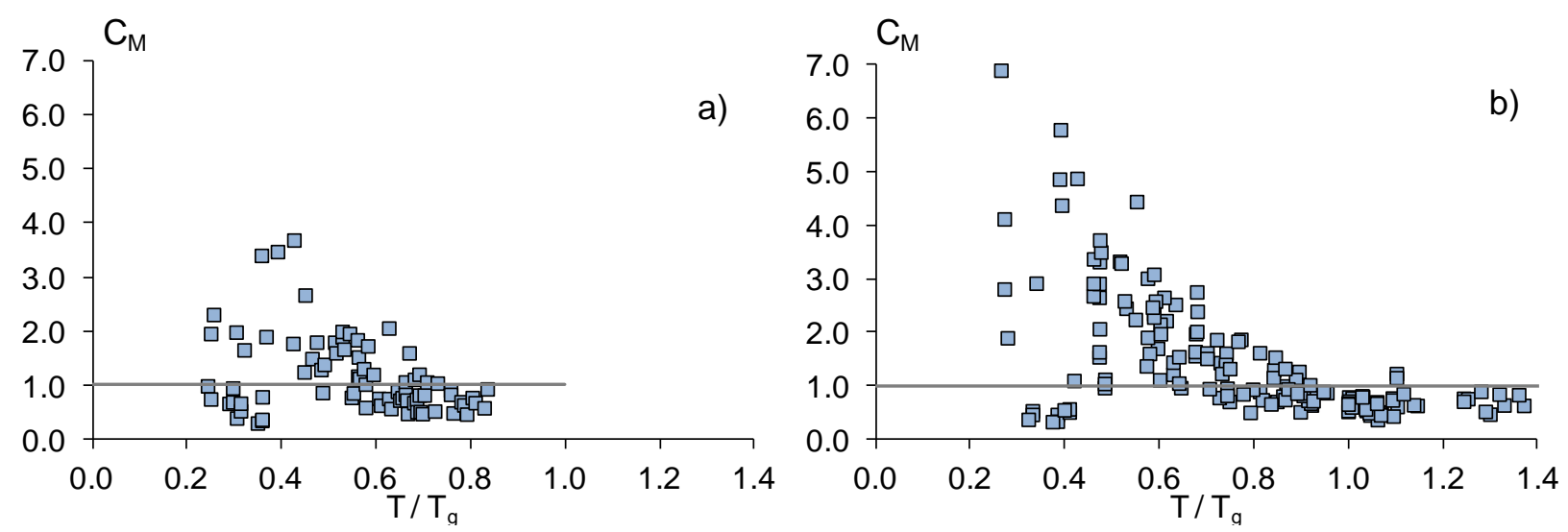

Figura 15. Cocientes del desplazamiento máximo inelástico y elástico de azotea, $C_{M}$, obtenidos para los dos conjuntos de edificios: a) edificios de acero, $\mathrm{y}$ b) edificios de concreto reforzado.

Para explicar las observaciones anteriores, se procedió a realizar una revisión detallada de las historias de desplazamiento elástico e inelástico ante registros seleccionados, así como de la respuesta histerética momento-curvatura en elementos seleccionados. Por ejemplo, en la figura 16 se muestra la historia de desplazamiento de azotea (panel superior), así como la historia de curvatura (panel inferior izquierdo) y la respuesta momento-curvatura (panel inferior derecho) correspondiente a tres casos de relaciones de periodos $T / T_{g}<0.75$ obtenidas para el modelo A-10N $\left(T_{l}=1.06 \mathrm{seg}\right)$. A partir de las figuras, se puede observar que en los tres casos los desplazamientos inelásticos son mayores que los desplazamientos elásticos. Esta situación ocurre cuando los desplazamientos inelásticos se separan de los desplazamientos elásticos después de la ocurrencia de un pulso lo suficientemente intenso en la historia de aceleraciones del terreno. Lo anterior implica que sí un pulso ocasiona que la estructura fluya (es decir, incursiona en el intervalo de comportamiento inelástico), esta permanecerá exhibiendo desplazamientos inelásticos en la misma dirección hasta que ocurra un pulso que induzca una fuerza de inercia opuesta que completaría el ciclo. Dado que $T_{g}$ es sensiblemente mayor que $T$ (es decir, cocientes de periodos $T / T_{g}$ pequeños implican que los periodos dominantes del terreno son sensiblemente mayores que el periodo fundamental de la estructura), el tiempo que permanece la estructura en el intervalo inelástico es proporcional al periodo $T_{g}$. Sí la fuerza de inercia opuesta no es suficiente para regresar a la estructura a su posición original, la estructura tenderá a experimentar desplazamientos residuales importantes, como se ilustra en las Figuras 16a y 16c. 

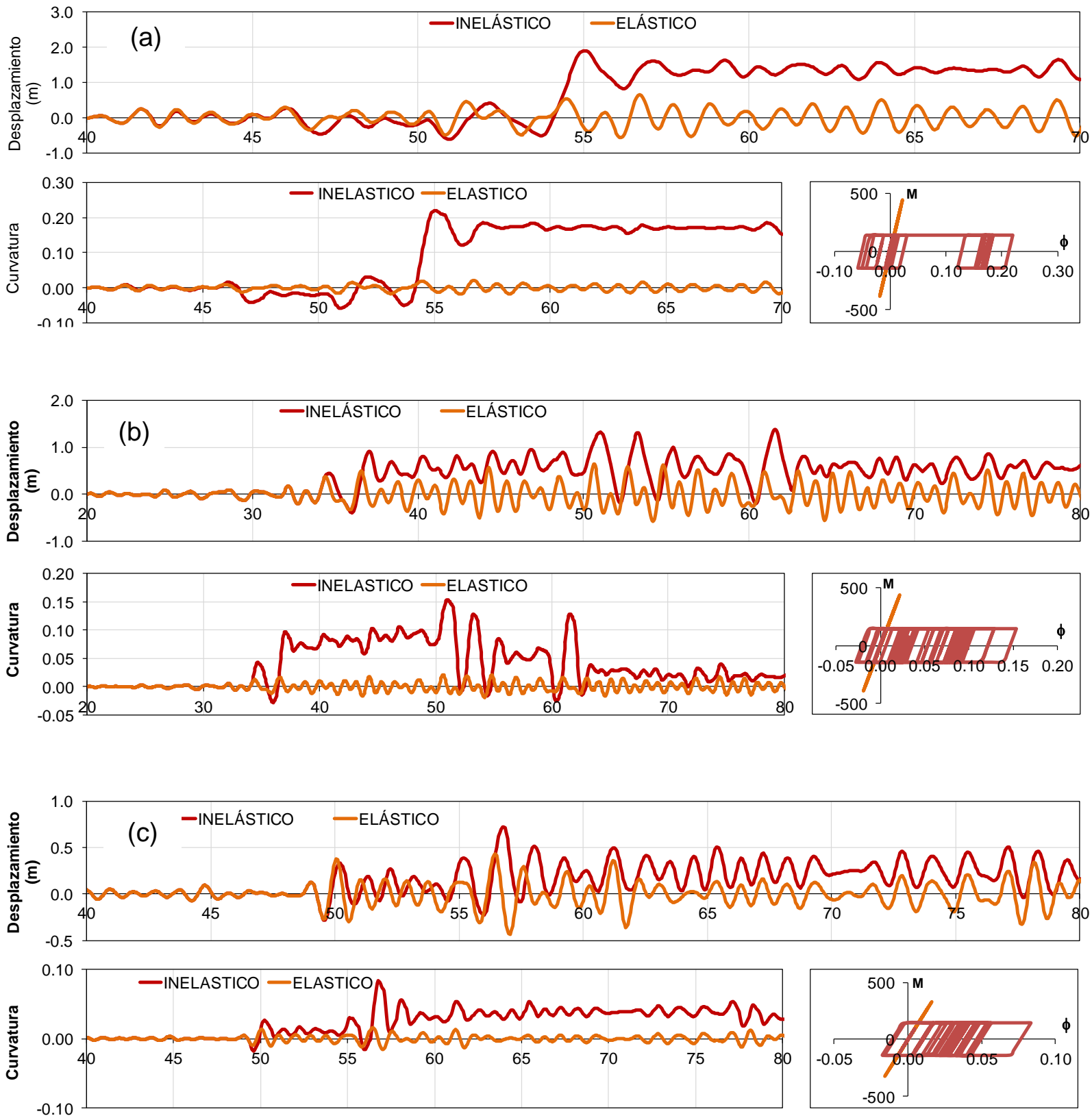

Figura 16. Historias de respuesta de desplazamientos (elástico e inelástico), curvatura y ciclo de histéresis: a) $T / T_{g}=0.37$, b) $T / T_{g}=0.51$, c) $T / T_{g}=0.59$.

A continuación, en la figura 17 se examinan algunos casos para el mismo edificio con cocientes de periodos $T / T_{g}$ mayores que 0.75 , pero menores que 1.55 . Cabe recordar que de acuerdo a lo observado en la Figura 15, en general, los desplazamientos inelásticos son menores que los desplazamientos elásticos. En esta zona espectral, las fuerzas de inercia generadas por la aceleración del terreno que actúan sobre la estructura tienen menor duración tanto en un sentido como en el otro, y por lo tanto, el tiempo de incursión en el intervalo inelástico es pequeño. Las fuerzas cíclicas no actúan el tiempo suficiente en la misma dirección para inducir en la estructura deformaciones grandes que puedan superar a las deformaciones que se generarían si el sistema se comportara elásticamente. Se puede ver que los ciclos histeréticos no se mueven lejos del origen, lo que implica que los desplazamientos residuales sean mínimos, o nulos, lo que 
contribuye a que la amplitud de los desplazamientos inelásticos sea menor a la amplitud de los desplazamientos elásticos.
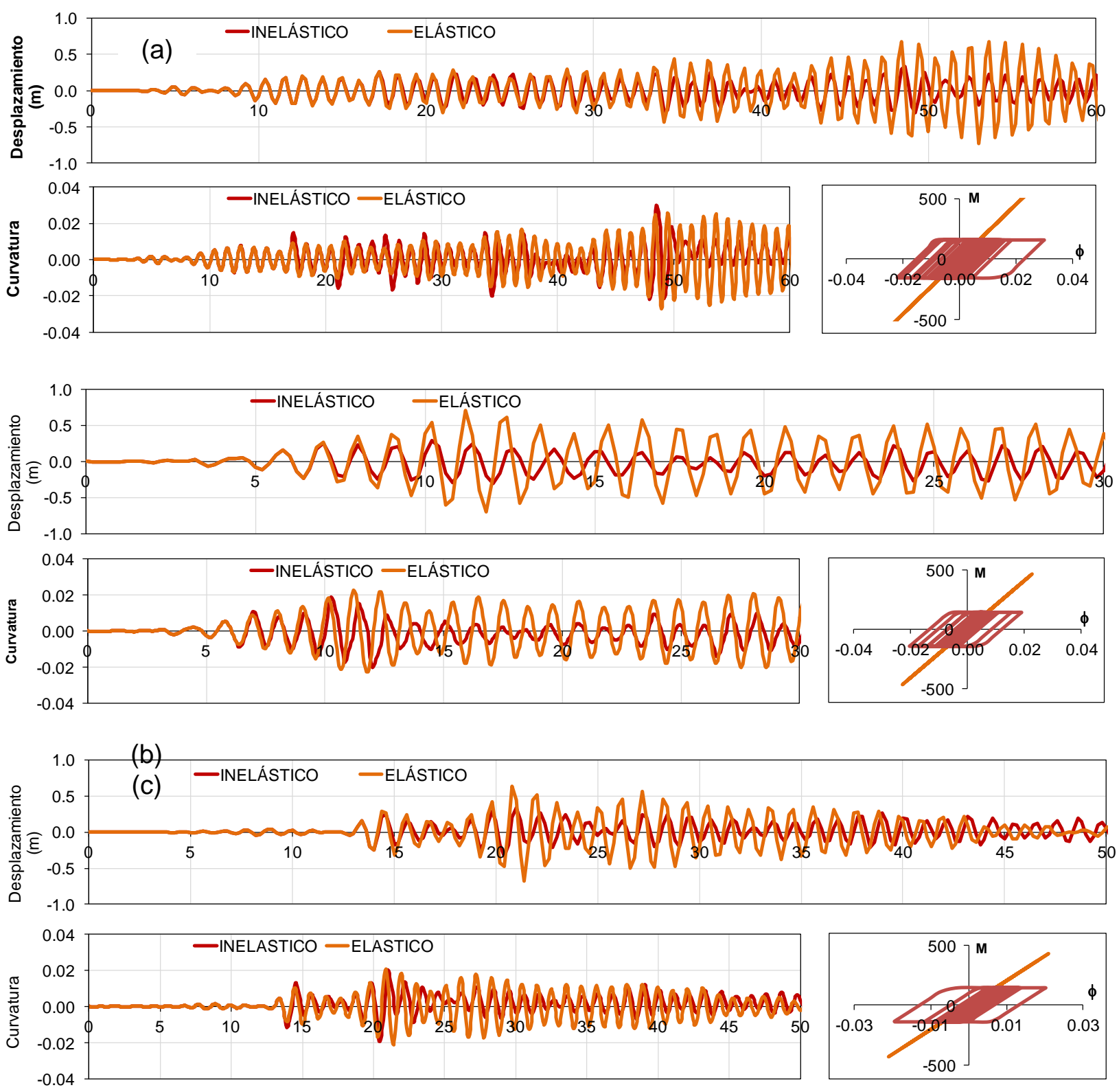

Figura 17. Historias de respuesta de desplazamientos (elástico e inelástico), curvatura y ciclos de histéresis: a) $T / T_{g}=0.76$, b) $T / T_{g}=0.79$, c) $T / T_{g}=0.83$.

\section{EVALUACIÓN DE DESPLAZAMIENTOS CON EL CRITERIO DE LAS NTCS-04}

Para evaluar los desplazamientos inelásticos con los criterios establecidos en las NTCS-04 en los edificios de acero, se realizó un análisis estático, el cual es comúnmente empleado en la práctica profesional. Las fuerzas sísmicas utilizadas para realizar dicho análisis se calcularon de acuerdo al criterio establecido 
en la sección 8 y fueron reducidas por el factor de reducción, $Q$ ', definido en la sección 4.1 del cuerpo de las NTCS-04 y por el producto del factor de reducción, $Q^{\prime}$, y de sobrerresistencia, $R$, en el caso del Apéndice A de las NTCS-04. Posteriormente, los desplazamientos laterales obtenidos del análisis estático se multiplicaron por los factores correspondientes, para condiciones de servicio y colapso mencionados en el Apéndice A. Con el criterio del cuerpo del reglamento, dichos desplazamientos se multiplicaron directamente por $Q$.

En la figura 18 se muestran tres ejemplos de los perfiles de desplazamiento del edificio de acero de 4 niveles ( $4 \mathrm{~N}-\mathrm{A}, \mathrm{T}_{1}=0.74 \mathrm{~s}$ ), con relaciones $T / T_{g}$ cercanas a 0.5 (es decir, donde $T_{g}$ es sensiblemente más largo que $T$ ), obtenidos con un análisis estático lineal (denominado "ESTÁTICO"), así como con los perfiles de desplazamiento inelástico aplicando los criterios tanto del cuerpo del reglamento (denominado "INELÁSTICO") como del Apéndice A (denominado "COLAPSO"). Asimismo, se indica el desplazamiento correspondiente a condiciones de servicio (denominado "SERVICIO"). Como puede apreciarse en las figuras, los criterios normativos indican que los desplazamientos inelásticos son mayores que lo desplazamientos obtenidos con el análisis estático lineal. Para verificar la tendencia observada, se incluyen los perfiles de desplazamientos obtenidos de un análisis dinámico empleando el programa RUAUMOKO (Carr, 2009) y considerando un sismo de servicio, denominados "S. SERVICIO". De acuerdo a Ordaz et al. (2003), un ejemplo de un sismo de servicio puede considerarse al evento ocurrido el 25 de abril de 1989 (con magnitud de onda superficial, Ms=6.9). Como puede observarse en las figuras, los desplazamientos asociados a sismos de servicio inducen desplazamientos laterales menores que los predichos por el cuerpo y el Apéndice A de la normatividad; sin embargo, se puede apreciar que existe una mejor aproximación cuando se considera el criterio para condiciones de servicio del Apéndice A. Para definir un sismo de "colapso" y poder verificar el criterio del Apéndice A, los acelerogramas se escalaron para alcanzar la velocidad máxima del terreno registrada en la estación SCT, componente EW, registrada durante el sismo del 19 de septiembre de 1985 (Terán-Gilmore y Jirsa, 2005). De esta manera, en las figuras se incluye la respuesta elástica, denominada "S. COLAPSO-ELÁSTICO", y a la respuesta inelástica, denominada "S.COLAPSO-INELASTICO". Al aplicar los criterios establecidos en el Apéndice A para predecir desplazamientos, se puede apreciar que el criterio para seguridad ante colapso tiende a sobreestimar significativamente los desplazamientos inelásticos. Asimismo, es de notar que los desplazamientos elásticos e inelásticos son muy similares, lo cual contradice el criterio de amplificación de desplazamientos incluido en la normatividad. Las observaciones anteriores fueron consistentes para otros edificios con relaciones $T / T_{g}$ similares.

En la figura 19 se muestran gráficas similares correspondientes a edificios de acero de 10 niveles (modelo A-10N, $T_{l}=1.06 \mathrm{seg}$ ) sometido a tres excitaciones con $T_{g}$ es cercano a $T$. Se puede observar que los desplazamientos obtenidos con el método estático sobre-estiman los desplazamientos laterales obtenidos para un sismo de servicio. Sin embargo, los "desplazamientos inelásticos" predichos por el cuerpo de las NTCS-04 son menores que los desplazamientos inelásticos calculados considerando los registros escalados para simular la condición de colapso. Por otra parte, puede notarse que los desplazamientos predichos con el criterio de condiciones de servicio del Apéndice A tienen una mejor aproximación a los desplazamientos calculados para los registros asociados al sismo de servicio. Asimismo, en las figuras se puede apreciar que los desplazamientos laterales predichos con el criterio de seguridad contra colapso del Apéndice A tienden a sobre-estimar los desplazamientos laterales inelásticos calculados con los registros escalados. Finalmente, es interesante notar que ante estos acelerogramas, los desplazamientos laterales elásticos son mayores que los desplazamientos laterales inelásticos, lo cual es consistente con la tendencia observada en este estudio. 

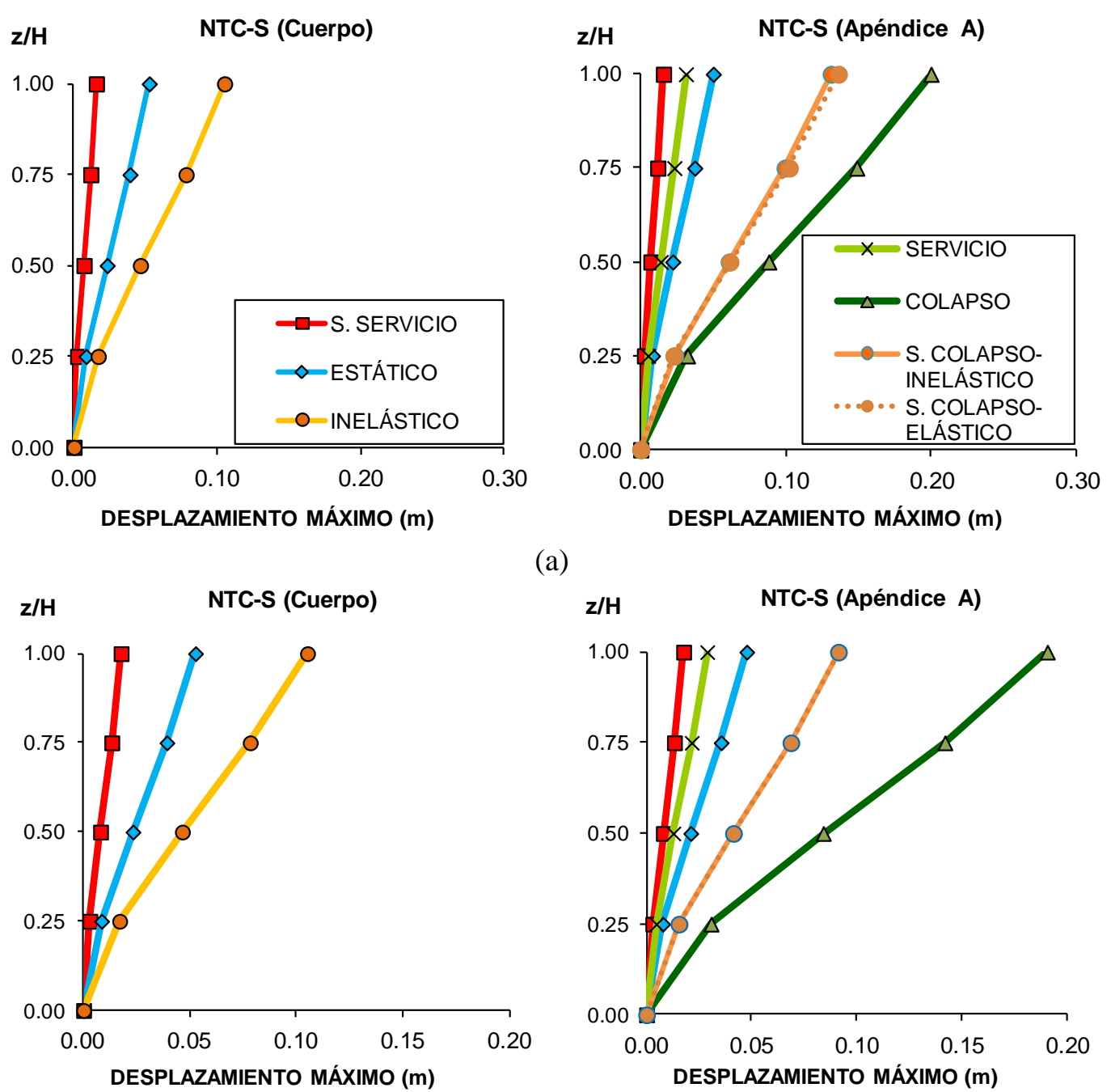

(a)

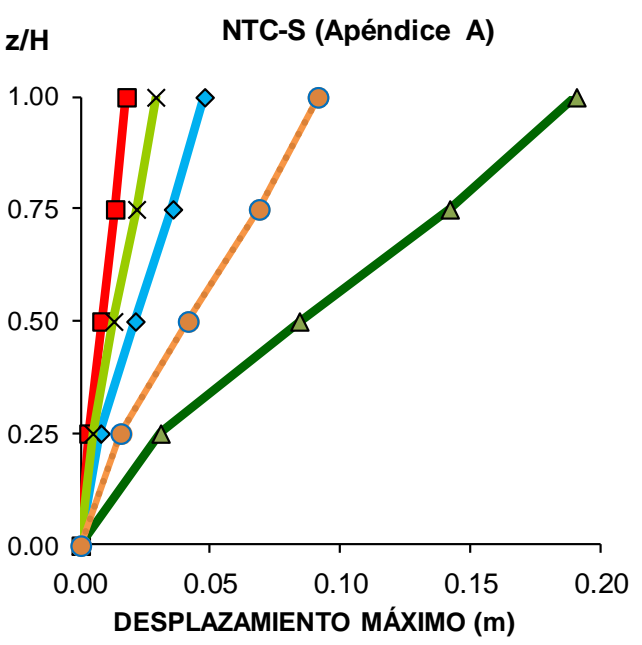

(b)
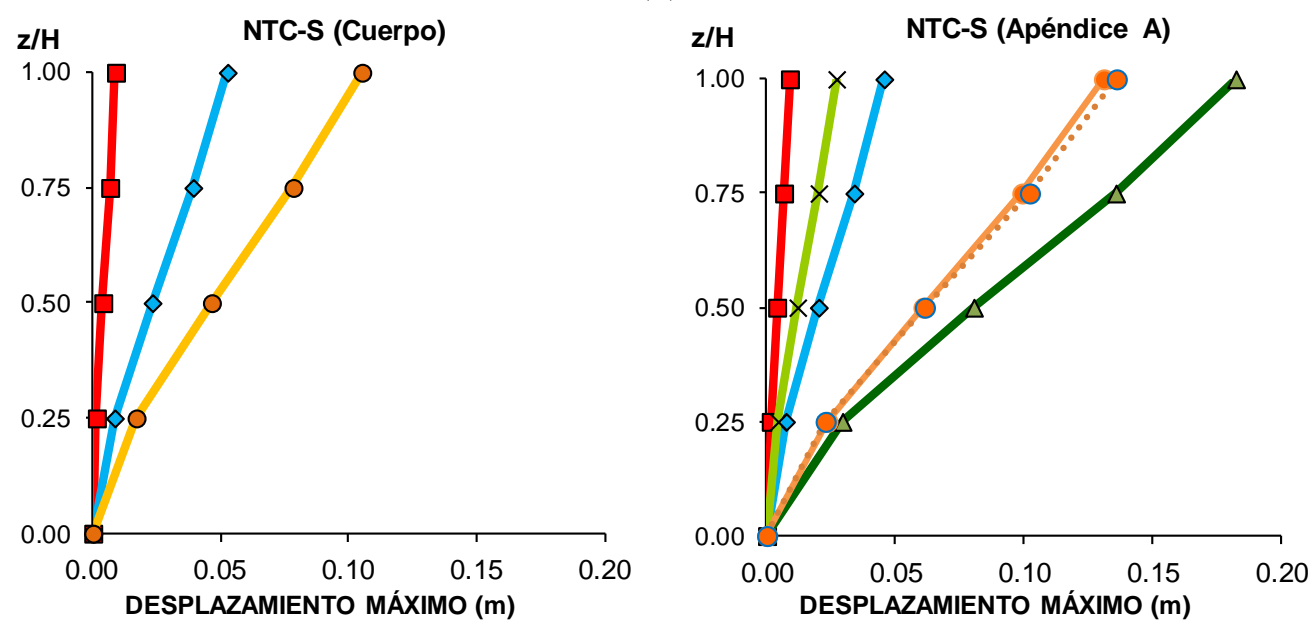

(c)

Figura 18. Perfiles de desplazamiento máximo elástico e inelástico obtenidos con los criterios del cuerpo y el Apéndice A de las NTCS-04 para $T / T_{g}<0.55$ : a) registro 5325ABEW, b) registro RO25ABNS, c) registro RO14SENS. 

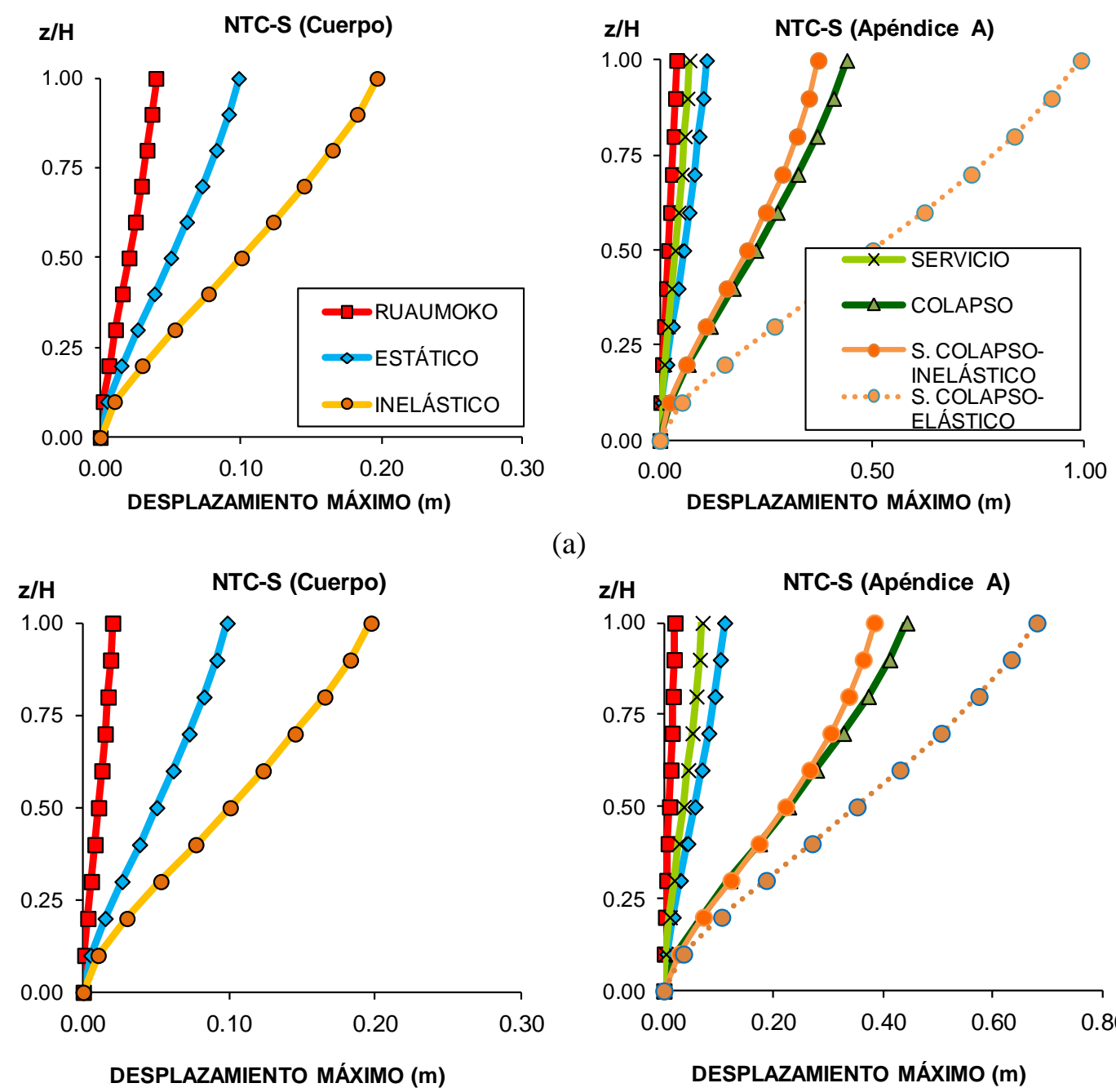

(a)

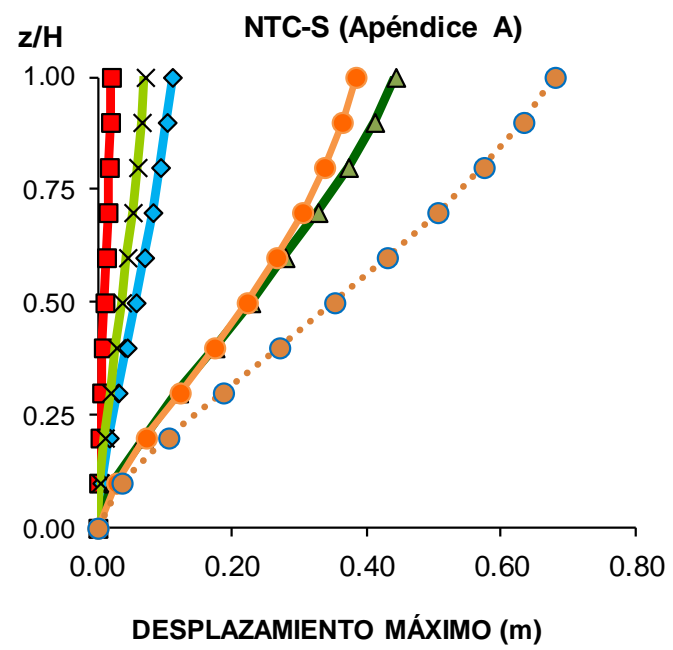

(b)
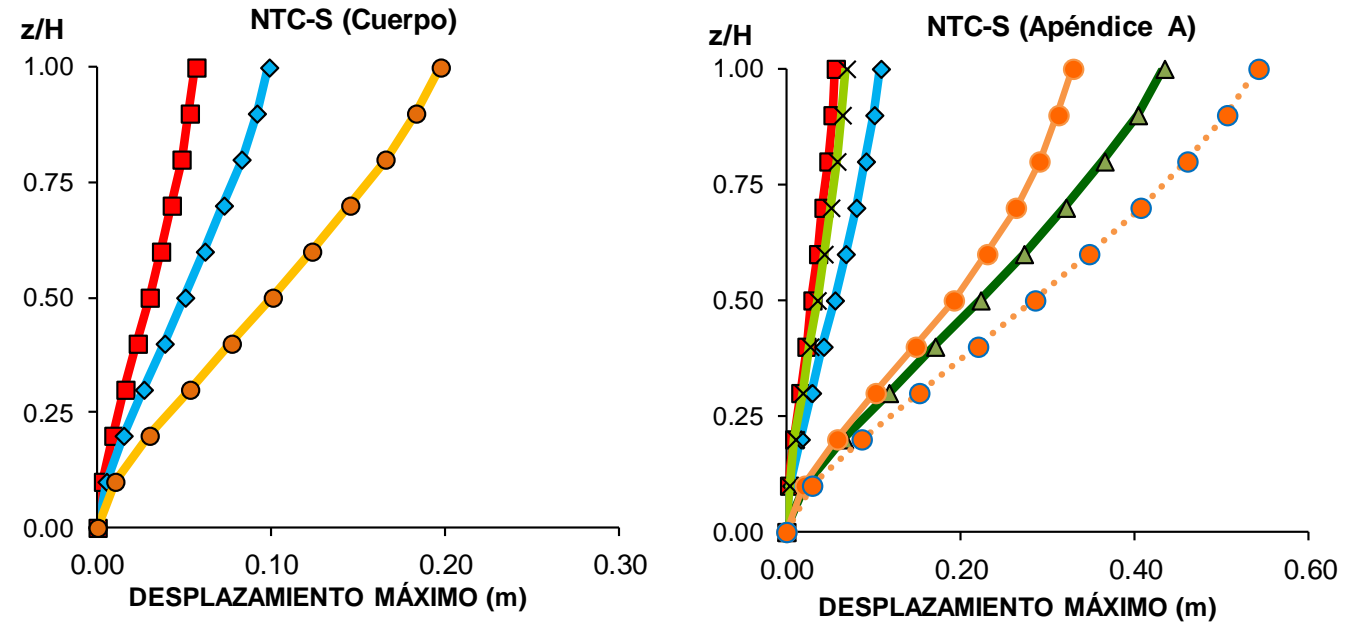

(c)

Figura 19. Perfiles de desplazamiento máximo elástico e inelástico obtenidos con los criterios del cuerpo y el Apéndice A de las NTCS-04 para $T / T_{g}$ cercanos a uno: a) registro 4424OCEW, b) 5524OCNS,

c) registro RO14SEEW. 


\section{CONCLUSIONES}

La estimación de las demandas de desplazamiento lateral que una estructura puede sufrir ante excitaciones sísmicas moderadas o intensas es una tarea muy importante en el diseño sismorresistente. Para tal fin, las Normas Técnicas Complementarias para el Diseño por Sismo en su edición 2004 (NTCS-04) proporcionan implícitamente dos criterios para estimar los desplazamientos laterales inelásticos. En esta investigación, se evalúo la aplicabilidad de dichos criterios con respecto a la tendencia observada de la respuesta dinámica elástica e inelástica de dos conjuntos de edificios estructurados a base de marcos ubicados en la zona de terreno blando de la Ciudad de México. A continuación, se ofrecen las siguientes conclusiones:

- A partir de los análisis dinámicos lineales y no-lineales de los edificios considerados en este estudio, se observa que existe una zona espectral donde los desplazamientos laterales elásticos son mayores que los desplazamientos laterales inelásticos. Lo anterior corrobora la tendencia observada en estudios previos que emplearon sistemas de un grado de libertad (Miranda, 1991; Santa Ana, 1996; Miranda y Ruiz, 1999; Espinoza y Terán, 2000; Ruiz-García y Miranda, 2004; Ruiz-García y Miranda, 2006).

- Los criterios normativos para estimar desplazamientos laterales indicados tanto en el Cuerpo como en el Apéndice A de la normatividad vigente no reconocen una zona espectral donde los desplazamientos laterales elásticos son mayores que los desplazamientos laterales inelásticos, lo cual puede conducir a errores importantes al estimar los desplazamiento laterales en edificios estructurados a base de marcos.

- El criterio para estimar desplazamientos laterales en condiciones de servicio propuesto en el Apéndice A proporciona una estimación razonable de los desplazamientos laterales producidos por registros que pueden considerarse como provenientes de sismos de servicio.

Con base en los resultados anteriores, es recomendable revisar los criterios establecidos en las 2004 (NTCS-04) para proponer criterios que permitan una estimación más razonable de los desplazamientos laterales y permitan considerar las tendencias identificadas en este estudio.

\section{AGRADECIMIENTOS}

Los autores quisieran agradecer las facilidades brindadas por la Universidad Michoacana de San Nicolás de Hidalgo para la realización de este trabajo, así como la generosidad académica del Dr. Amador Terán Gilmore por facilitar la información necesaria para modelar los edificios de concreto empleados en este estudio. Asimismo, el segundo autor expresa su agradecimiento al Consejo Nacional de Ciencia y Tecnología por el apoyo económico brindado para cursar sus estudios de maestría. Finalmente, los autores agradecen los comentarios de dos revisores anónimos que permitieron mejorar el contenido del artículo que se presenta.

\section{REFERENCIAS}

Bazán, E y R Meli (1999), Diseño Sísmico de Edificios, Editorial Limusa, 2a . Reimpresión, México.

Carr, A J (2009), "RUAUMOKO2D-Inelastic Dynamic Analysis Program. User manual for the 2Dimensional version", Dept. of Civil Engineering, University of Canterbury, Christchurch, New Zealand. 
Carrillo, J, H Hernández y A Rubiano (2014), "Analysis of earthquake-resistant design approach for buildings in Mexico", Ingeniería Investigación y Tecnología, volumen XV (número 1): 151-162.

Espinoza, M A y A Terán (2000), "Efecto de la degradación de rigidez en las demandas sísmicas de sistemas simples”, Memorias XII Congreso Nacional de Ingeniería Estructural, León, CDROM.

Díaz, M A (2006), “Confiabilidad sísmica de edificios de acero diseñados con el RCDF-2004”, Tesis de Maestría, Universidad Nacional Autónoma de México; 69 pp., México. http://www.ptolomeo.unam.mx:8080/xmlui/bitstream/handle/132.248.52.100/892/DIAZGONZALE Z.pdf?sequence $=1$

Gaceta Oficial del Departamento del Distrito Federal (1997), Reglamento de Construcciones para el Distrito Federal, Ciudad de México.

Gaceta Oficial del Distrito Federal (2004), Reglamento de Construcciones para el Distrito Federal, 29 de enero del 2004, Ciudad de México.

Ordaz, M., E Miranda y J Avilés (2003), "Propuesta de espectros de diseño por sismo para el D.F.", Revista Internacional de Ingeniería de Estructuras, 8 (2): 189-207.

Miranda, E (1991), "Seismic evaluation and upgrading of existing buildings", Ph.D. Thesis, University of California, Berkeley.

Miranda, E y J Ruiz (1999), "Influencia de la degradación de rigidez en las demandas de desplazamiento lateral de estructuras cimentadas en suelos blandos", Memorias XII Congreso Nacional de Ingeniería Sísmica, Morelia.

NTCS-04 (2004), Normas Técnicas Complementarias para Diseño por Sismo, Reglamento de Construcciones para el Distrito Federal. Gaceta Oficial del Distrito Federal.

Ruiz-García, J y E Miranda (2004), "Inelastic displacement ratios for design of structures on soft sites", Journal of Structural Engineering ASCE, 130 (12), 2051-2061.

Ruiz-García, J y E Miranda (2006), "Inelastic displacement ratios for evaluation of structures built on soft soils sites," Earthquake Engineering and Structural Dynamics, 35(6): 679-694.

Santa Ana, P R (1996), "Demandas de desplazamiento inelástico en estructuras en la zona blanda de la ciudad de México", Tesis de Maestría, Universidad Nacional Autónoma de México; 104 pp. México D.F., México.

Sociedad Mexicana de Ingeniería Sísmica (1999). Base Mexicana de Datos de Sismos Fuertes, Catálogo de Acelerogramas 1960-1999 CD.

Seismosignal (2016). Earthquake Engineering Software Solutions. http://www.seismosoft.com/seismosignal.

Terán, A (1998), "Características mecánicas y desempeño sísmico de marcos dúctiles de concreto reforzado", Memorias del XI Congreso Nacional de Ingeniería Estructural, Monterrey, NL, México.

Teran-Gilmore, A (2004), "On the use of spectra to establish damage control in regular frames during global predesign”, Earthquake Spectra, 20(3):995-1020. 
Teran-Gilmore, A y J O Jirsa (2005), "A damage model for practical seismic design that accounts for low cycle fatigue”, Earthquake Spectra, 21(3): 803-832.

Uang, C-M y M Maarouf (1994), "Deflection amplificaction factors for seismic design provisions", Journal of Structural Engineering ASCE, 130(8): 2423-2446.

Uang, C-M (1989), "Establishing R (or Rw) and Cd Factors for Building Seismic Provisions", Journal of Structural Engineering ASCE, 117 (1): 19-28. 